\title{
Major projects in urban sites: Challenge of the Builders
}

\section{Grands projets en site urbain: Défi et chalenge des Bâtisseurs}

\section{Ammar DHOUIB}

\author{
VINCI, Paris France \\ 61, avenue Jules Quentin 92730 Nanterre cedex. Courriel : ammar.dhouib@ vinci-construction.fr
}

\begin{abstract}
Faced with the complexity of urban construction projects and difficulties in the field, engineers must, on the one hand, dimension with rigor and common sense the foundations with all the requirements of today of quality, conformity and respect of deadlines and budget and take into account, on the other hand, safety and environmental requirements and societal and sustainable development criteria, the purpose of this communication is to present concrete projects of foundations and excavation deep in geologically heterogeneous and highly urbanized sites, with monitoring and displacement measurements in order to compare predictions with reality and to promote the "observational method".
\end{abstract}

\begin{abstract}
Résumé. Face à la complexité des projets de construction en site urbain et aux difficultés de terrain, les ingénieurs doivent, d'une part, dimensionner avec rigueur et bon sens les fondations avec toutes les exigences d'aujourd'hui de qualité, de conformité et de respect de délais et de budget et tenir compte, d'autre part, des impératifs de sécurité et d'environnement et des critères sociétaux et de développement durable, L'objectif de cette communication est de présenter des projets concrets de fondations et d'excavation profondes dans des sites géologiquement hétérogènes et fortement urbanisés, avec suivi et mesures des déplacements afin de confronter les prédictions à la réalité et de promouvoir la « méthode observationnelle ».
\end{abstract}

\section{Préambule}

Les projets sont de plus en plus complexes entre tours de plusieurs dizaines, voire plusieurs centaines de mètres de hauteur parfois implantées au milieu d'ouvrages existants sensibles, excavations profondes, ponts en site aquatique et tunnels dans des sols meubles et en milieux urbains sensibles.

A ce défi affronté par les bâtisseurs s'ajoutent les problèmes de terrain où les bons terrains sont, de nos jours, rares. Car, entre friches industrielles et terrains marécageux, le sol est souvent aquifère, mou et/ou compressible et ses déformations sous le poids des ouvrages sont importantes et deviennent, dans ce contexte, souvent incompatibles avec les déplacements admissibles de la structure.

Face à La réglementation, qui a connu un développement considérable et devient de plus en plus exigeante par de nouvelles normes françaises et européennes: missions géotechniques normalisées et
« Eurocodes », se limiter à dimensionner, avec rigueur et bon sens, les fondations ne suffit plus en raison, d'une part, des exigences d'aujourd'hui de qualité, de conformité et de respect de délais et de budget et, d'autre part, des impératifs de sécurité et d'environnement et des critères sociétaux et de développement durable.

L'objectif de cette communication est de présenter des projets concrets de fondations d'une grande tour dont le noyau central apporte plus de $1.100 .000 \mathrm{kN}$ à un radier général assis sur substratum en forte pente, de fondations superficielles sur semelles, de pieux sous charges verticales centrées, et enfin d'une grande excavation de $70 \mathrm{~m}$ de haut en site géologiquement hétérogène et fortement urbanisé.

Ces cas réels présentés ici ont fait l'objet de suivi et de mesures des déplacements, ce qui a permis de confronter les prédictions à la réalité du terrain et de « promouvoir » la « Méthode observationnelle », en guise de démontrer que les aléas et risques sont omniprésents en géotechnique, mais il est possible de les maitriser par 
des études bien faites, des exécutions soignées et des supervisions adaptées au sens de la norme NF P94-500.

\section{Cas de méga-radier d'une grande tour}

Dans le cadre d'un projet atypique d'une grande tour d'une quarantaine d'étages sous forme d'amende à façades complexes et lourdes et avec des charges fortement centrées sur un noyau central, le sol est complexe avec un substratum marneux parfois grésifié très compact plongeant de 2,00 à $-14,50$ NGF vers la mer et partiellement rempli de vases alluvionnaires issues des chenaux qui sillonnent les reliefs montagneux du site et se déversent dans la mer.

Sur le plan mécanique, l'analyse géotechnique initiale d'avant-projet (Mission G12), basée sur les essais pressiométriques, offrait un module pressiométrique moyen $\left(\mathrm{E}_{\mathrm{M}}\right)$ de $180 \mathrm{MPa}$ dans le substratum marneux. Des études complémentaires en phase d'exécution (Mission G3) avec des essais «haute pression » et des calibrages des sondes pour les corrections des modules pressiométriques selon les règles de la norme NF P94110 (Dhouib et al., 2002, Dhouib, 2016) ont abouti à un module pressiométrique corrigé moyen dans le substratum marneux de $415 \mathrm{MPa}$; ces mesures pressiométriques ont été accompagnées d'essais de compression simple avec mesures des modules de déformation $\mathrm{E}$ dans plusieurs échantillons marneux. Les essais complémentaires ont permis de valider en phase EXE une solution de fondations « mixte » :

- Radier général pour le noyau central de la tour, radier fondé équitablement sur le substratum et sur du gros béton remplaçant la vase alluvionnaire, le tout à l'abri d'une paroi de confinement à l'intérieur de la paroi périphérique principale,

- Appuis simples en fondations superficielles très ponctuels aux alentours, et,

- «Barrettes-raidisseurs » contre la paroi périphérique à la fois porteuse et faisant l'office d'enceinte étanche.

La figure 1.a illustre la coupe lithologique issue d'une série de sondages carottés, pressiométriques et destructifs enregistrés ayant permis de déceler avec précision (avec une maille fine de $25 \mathrm{~m}^{2}$ ) le toit du substratum marneux. Sur la figure 1.b est présentée l'emprise (en forme d'amende) de la tour avec toit des marnes plongeant vers la mer et position du noyau fondé sur le radier général.

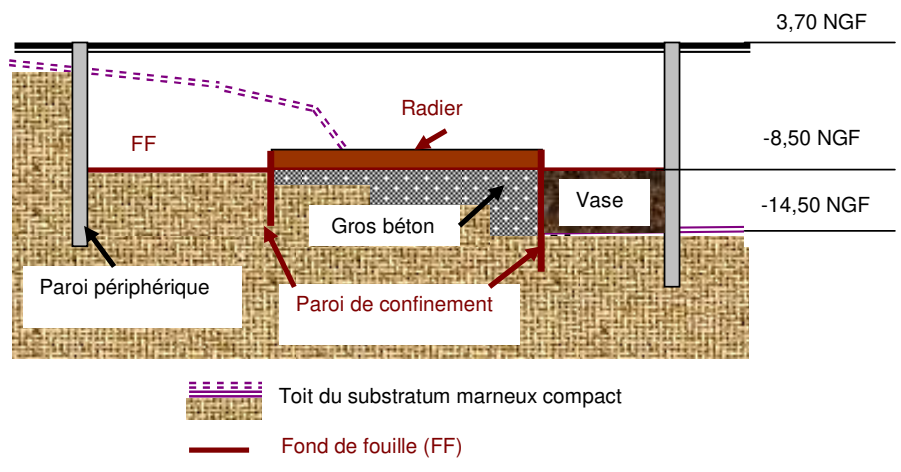

a. Coupe lithologique et fondation de la tour

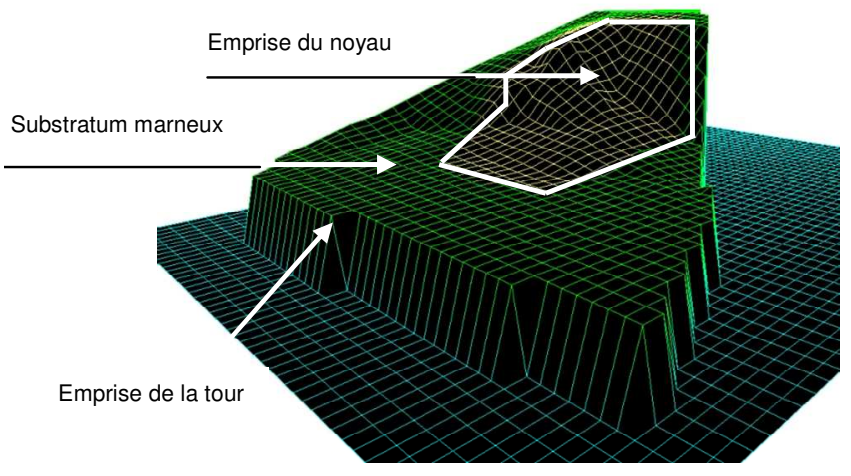

b. Emprise de la tour et position du noyau de la tour

Figure 1 : Contexte géotechnique du site et fondation du noyau de la tour

La figure 2.a met en évidence la synthèse des données pressiométriques $\mathrm{E}_{\mathrm{M}}$ corrigés à partir du calibrage des sondes (Dhouib et al., 2002, Dhouib 2016) et des pressions limites nettes $\mathrm{p}_{1}^{*}$ obtenus par des sondes « rocher » où les pressions ont été poussées jusqu'à 10 $\mathrm{MPa}$.

Les tassements de la tour ont été suivis sur une période de 19 mois sur plusieurs cibles, tassements que résume la figure 2.b pour quatre cibles disposées sur la périphérie du noyau en comparaison avec les tassements prédits par des calculs analytiques et numériques en éléments finis en deux dimensions.

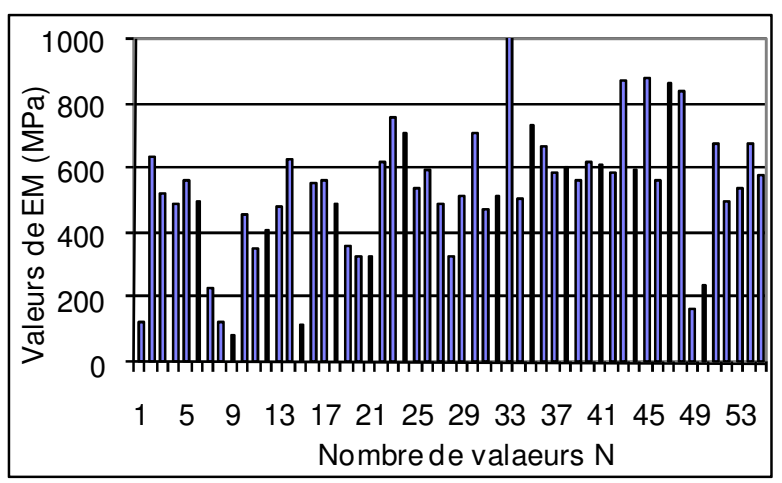

Moyenne harmonique $\mathrm{E}_{\mathrm{M}}=415 \mathrm{MPa}$

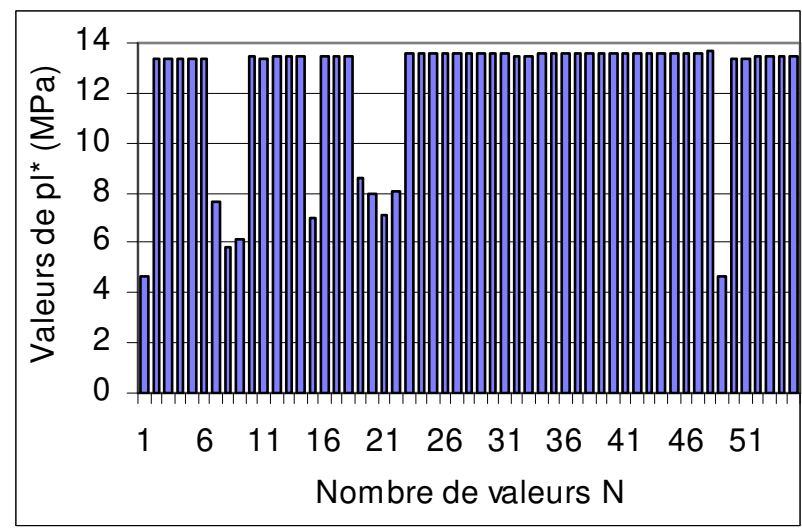


a. Synthèse des données pressiométriques

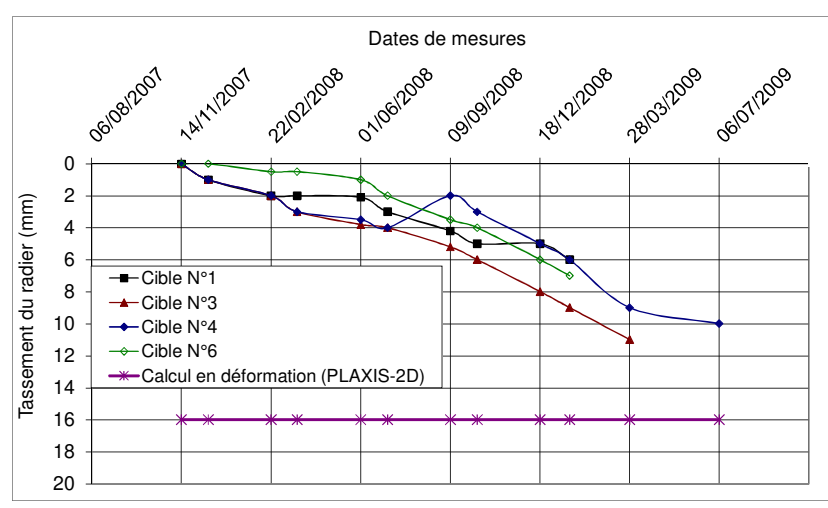

b. Tassements mesurés et prédits du radier général

Figure 2 : Données mécaniques et tassement du radier général de la tour

\section{Commentaires}

- Compte tenu du pendage du substratum, la tour a fait l'objet d'une vérification de la stabilité vis-àvis du glissement généralisé. Les coefficients de sécurité au glissement vont, selon les combinaisons des charges, de 2,10 à 7,10.

- La stabilité du fond de fouille est vérifiée vis-àvis du "renard-solide» avec un coefficient de sécurité de 16,1.

\section{Cas de semelles filantes}

Ce projet de bâtiment de faible hauteur de type $\mathrm{RDC}+3$ étages sur fondations superficielles filantes a fait l'objet de plusieurs études dans le cadre des missions géotechniques normalisées. La mission, G3 diligentée par l'entreprise en phase EXE, a été clôturée par un rapport de mission G3 - Phase étude.

Pour une emprise au sol du bâtiment de $1800 \mathrm{~m}^{2}, 3$ sondages initiaux (SP1, SP2 et SP3) dans la cadre des misions G1 et G2 ont été complétés en phase G3 par 2 sondages SP101 et SP102, afin de mieux couvrir l'emprise et respecter le ratio habituel de 1 sondage pour $400 \mathrm{~m}^{2}$ dans des sols sans anomalies particulières d'origine anthropique (Carrières, marnières, catiches) ou naturelle (dissolutions de gypse, poches karstique). Lorsque le sous-sol du projet se situe dans une zone à anomalies, les sondages doivent être plus serrés jusqu'à un sondage tous les $200 \mathrm{~m}^{2}$ (selon la notice de l'Inspection Générale des Carrières : IGC, 2003 en région parisienne), voire moins.

A partir des 5 sondages pressiométriques, la synthèse des contraintes aux Etats Limites Ultimes ELU et aux Etats Limites de Service ELS est, par application du DTU 13.12 (1992) pour le dimensionnement et la justification des semelles superficielles ancrées dans des formations de pente (éboulis) sableuses, est portée sur la figure 3.

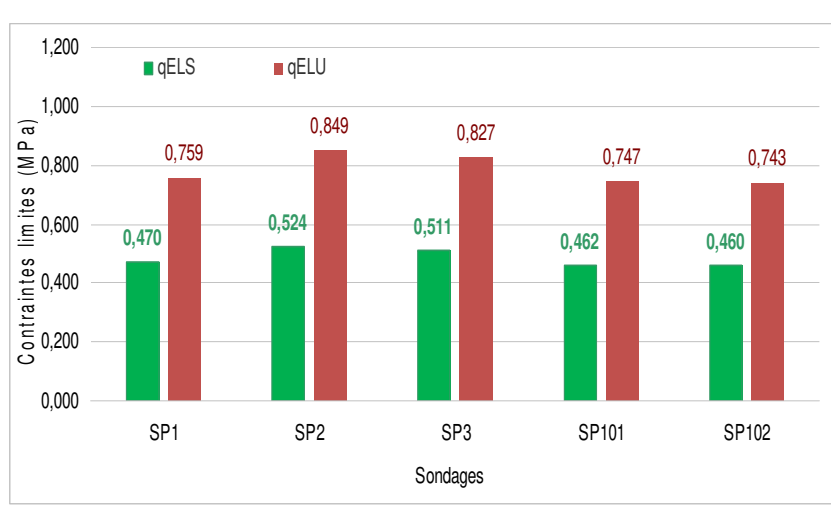

Figure 3 : Contraintes aux Etats Limites ( $\mathrm{q}_{\mathrm{ELU}}$ et $\mathrm{q}_{\mathrm{ELS}}$ )

La contrainte aux Etats Limites de Service ELS varie, d'après les histogrammes de la figure 3 , de 0,460 à 0,524 $\mathrm{MPa}$, ce qui permet de retenir raisonnablement une valeur de 0,450 MPa, alors que le rapport initial de mission G1 puis G2 la fixait arbitrairement à 0,30 MPa.

Sous la contrainte aux Etats Limites de Service de 0,450 MPa, les tassements théoriques absolus sont estimés entre $5 \mathrm{~mm}$ pour les semelles périphériques moins chargées (SF1 et SF2 de largeur 0,6 à 0,7 m) et 10 $\mathrm{mm}$ pour les semelles intérieures plus chargées (SF10 et SF11 de largeur 1,0 à 1,2 m), comme l'illustrent les graphes de la figure 4.a, qui comparent les tassements théoriques absolus aux tassements mesurés pour les semelles d'angle, dont les tassements ont été régulièrement suivis par de simples visées de géomètre pendant 24 mois.

Les tassements différentiels, selon une coupe type et représentative, ne dépassant pas les $0,5 \mathrm{~mm}$ entre files espacées de $6,0 \mathrm{~m}$, vérifient largement la règle usuelle de $1 / 500$, ce qu'illustre la figure 4.b.

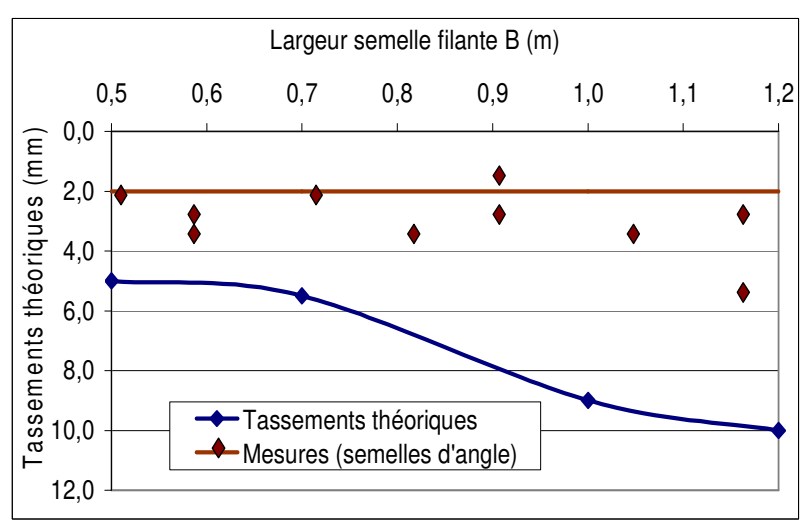

a. Tassements absolus : Mesures et prédiction 


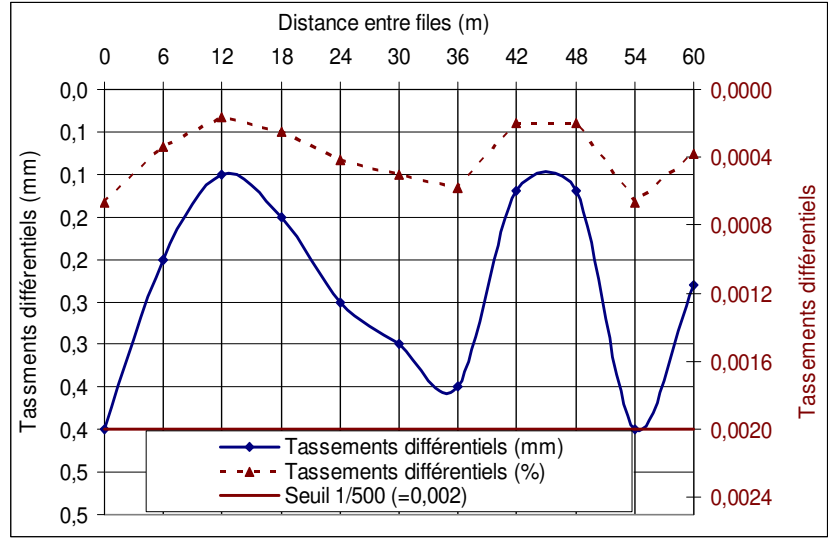

b. Tassements différentiels

Figure 4 : Tassements absolus et différentiels de semelles filantes

\section{Commentaires :}

- Les tassements théoriques absolus calculés ici sont faibles et vérifient parfaitement la règle TO de Ménard adaptée en très petites déformations (Baguelin et al., 2009). Ils sont compatibles avec la structure, et la contrainte aux Etats Limites de Service ELS de 0,450 MPa est donc admissible.

- Lorsque les essais pressiométriques sont bien faits (forage bien calibré) et les charges d'un ouvrage bien réparties entre files, respectant les distances usuelles et les fouilles bien soignées et curées, les tassements, totaux et différentiels, sont généralement faibles et toujours compatibles avec la structure; c'est ce qui fait la «force » de la méthode pressiométrique de Louis Ménard et son avantage par rapport aux autres méthodes de dimensionnement des fondations.

\section{Comportement de pieux sous charge axiale centrée}

Les pieux sous charge verticale ont fait l'objet, par le réseau des laboratoires des Ponts et Chaussées, de plusieurs instrumentations et prédictions des tassements depuis des décennies. Sur la figure 5 sont portés les tassements mesurés en tête de pieux ancrées dans les alluvions de la Seine de la région parisienne et soumis à une charge verticale centrée en comparaison avec les calculs en déformation (Eléments finis) et calculs basés sur le principe des fonctions de transfert (à l'aide du logiciel « PIVER ».

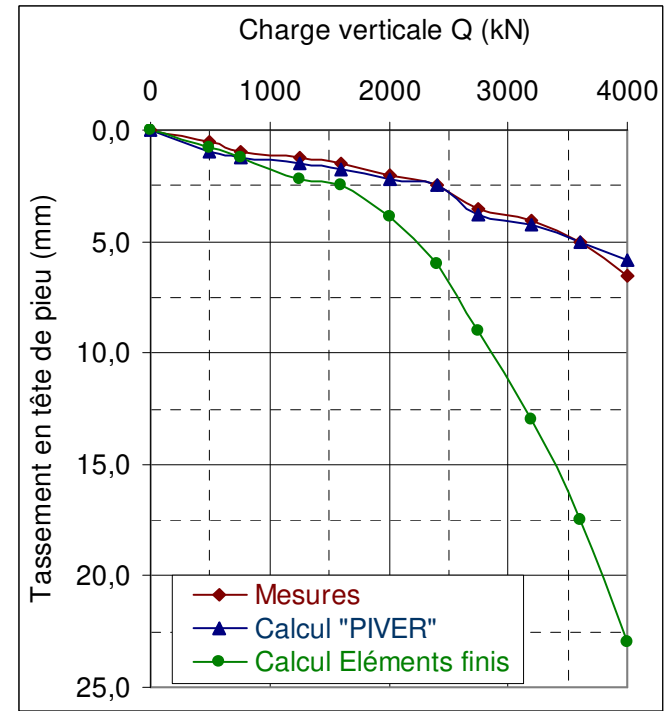

a. Pieu diamètre $650 \mathrm{~mm}$

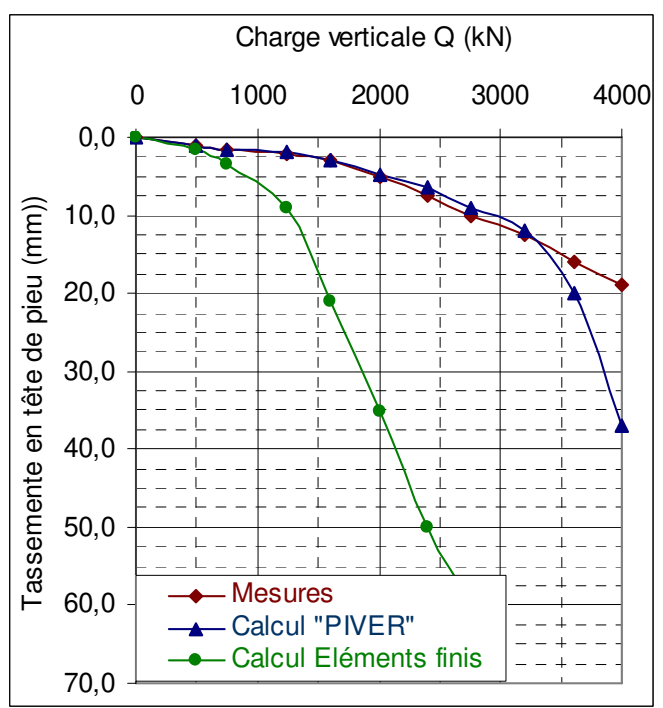

b. Pieux diamètre $1500 \mathrm{~mm}$

Figure 5 : Tassements en tête de pieux :

Mesures et prédictions

Notons que, dans les deux cas de pieux de la figure 5, les calculs en déformation par la méthode des éléments finis donnent des tassements beaucoup plus importants que les enfoncements mesurés en têtes de pieux, et ce notamment pour les charges importantes. Ceci est inhérent aux calculs en déformation qui font appel à des modules de déformation, soit mal mesurés, soit mal appréciés. Un autre inconvénient des modèles en éléments finis réside dans la mobilisation de déformations dans les couches profondes qui normalement ne tassent pas. Les calculs utilisant les fonctions de transfert offrent, dans les deux cas de pieux, un parfait ajustement, à l'exception des charges extrêmes pour le fût de $1500 \mathrm{~mm}$ de diamètre.

\section{Retour d'expérience et leçons à tirer}


Les différents cas réels d'ouvrages présentés dans cette présentation montrent que les tassements mesurés sont toujours inférieurs aux prédictions issues des calculs, qu'ils soient analytiques ou numériques. La tendance des jeunes ingénieurs est de courir, de nos jours, aux calculs numériques car il est plus facile d'allumer son ordinateur et de lancer une «boite noire» que constituent notamment les codes en éléments finis que de prendre son crayon, du papier et la calculatrice pour avoir au moins l'ordre de grandeur des phénomènes.

Pour rendre plus crédible leur action en géotechnique, les ingénieurs doivent d'abord analyser avec critique et attention les données issues des mesures, qu'elles soient sur site ou en laboratoire, en faire une synthèse et les vérifier par rapport à des données existantes pour retenir un jeu ou plusieurs jeux de paramètres avant de se lancer dans les calculs. Lorsque les prédictions sont faites, il est important et primordial d'élaborer un vrai programme de mesures et de suivi préalable sur chantier et le mettre en exécution dès le démarrage du chantier. Ce qui n'est pas toujours facile même dans des grandes entreprises de renom car les maîtres de l'ouvrage ne veulent pas aller dans ce sens pour raisons d'économie.

Afin d'enrichir ce retour d'expérience, nous avons apporté sur la figure 6 les tassements mesurés et calculés sous plusieurs ouvrages réels, allant des semelles superficielles, en passant par les radiers généraux, jusqu'aux remblais routiers et autoroutiers.

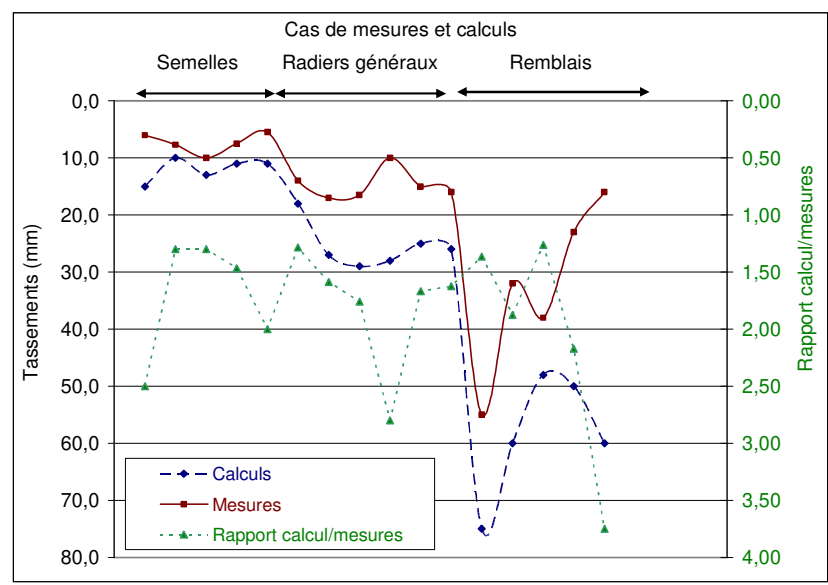

Figure 6 : Exemples de tassements mesurés et calculés sur ouvrages réels

A l'examen des graphes de la figure 6, il y a lieu de noter :

Les tassements théoriques sont toujours supérieurs aux tassements mesurés. Le rapport des tassements calculés aux tassements mesurés varie de 1,14 (un cas de semelles superficielles) à 3,75 (un exemple de remblai sur sol moyennement compact).

- Sont rares les cas où les tassements mesurés dépassent les tassements prédits par les calculs. Mais il y a, parfois, des exemples atypiques : un remblai de faible hauteur repose sur une puissance de $3 \mathrm{~m}$ de limons où divers essais ont été effectués le mois de juillet en plein été. Les calculs des tassements ont été conduits sur la base de caractéristiques mécaniques «estivales » dans des limons secs qui sont trois fois plus élevées que les valeurs réelles «hivernales » dans les limons humides avec des battements de la nappe en relation directe avec un ruisseau tout proche. Les tassements mesurés étaient trois plus importants que les tassements calculés. Un autre cas de remblai de $6 \mathrm{~m}$ de haut sur une décharge de $12 \mathrm{~m}$ « améliorée » par des inclusions rigides a conduit à des tassements presque 5 fois plus élevés que ceux calculés à partir de mauvais rentrants en raison du caractère très hétérogène des matériaux de la décharge et de points singuliers en termes de modules pressiométriques.

- Dans les divers projets instrumentés et suivis par nos soins ou apportés à notre connaissance par la profession et constatés dans les expertises judiciaires et extrajudiciaires, le rapport des tassements calculés aux mesurés sous fondations (semelles superficielles, radiers et remblais) et entre déplacements calculés et mesurés (écrans et murs de soutènement) de 1,5 à 3 est une valeur courante en géotechnique.

- En ce qui concerne les fondations profondes sur pieux, barrettes et parois porteuses, les enfoncements des fûts dans le sol sont faibles, voire nuls car la pointe des pieux est généralement toujours ancrée (ancrage effectif) dans un sol résistant et compact. Dans ce cas, les tassements des pieux se réduisent aux raccourcissements élastiques sur la hauteur de la couche médiocre. Cependant, il y a eu bel et bien quelques cas, mais rares, de sinistres liés aux tassements excessifs de pieux métalliques ouverts ancrés par battage dans la craie qui se «liquéfie» ( Phénomène de thixotropie ») en cours de battage et de pieux particuliers forés à la tarière, non suffisamment ancrés dans le substratum marneux compact et ayant conduit à un sinistre très couteux en matériel (travaux lourds de reprise par micropieux autour des pieux défaillants) et immatériel (bâtiment non livré à temps et personnel déplacé sur un autre site).

- Les tassements des fondations d'un ouvrage dépendent à la fois de la compacité du sol support et de la contrainte qu'apporte au sol la fondation de l'ouvrage. En fait, les deux paramètres sont liés, car une tour de grande hauteur nécessite un support résistant pour être fondée alors qu'un pavillon a besoin d'un «petit bar $(0,1 \mathrm{MPa})$ » pour asseoir ses fondations dans un limon (la « terre du paysan »).

Sous les contraintes appliquées par l'ouvrage, en partant d'un état initial de faible niveau de déformation caractérisé par un module de déformation du sol maximal $\mathrm{E}_{0}$ (soit un module de cisaillement maximal $\mathrm{G}_{0}$ ), le sol se déforme et sa rigidité diminue lorsque la déformation $(\varepsilon)$ sous l'ouvrage croît. Le comportement des ouvrages de construction (fondations, murs et écrans de soutènement, tunnels souterrains, remblais sur sol compressible) et leur stabilité et pérennité dans le temps dépendent du niveau 
de déformation atteint pendant l'application de la sollicitation

La figure 7 présente le domaine conventionnel du niveau de déformation que peut accepter les ouvrages de construction et les variations des modules de déformation $\left(E_{s}\right)$ et de cisaillement $\left(G_{s}\right)$ du sol sous ces ouvrages en fonction de la déformation.

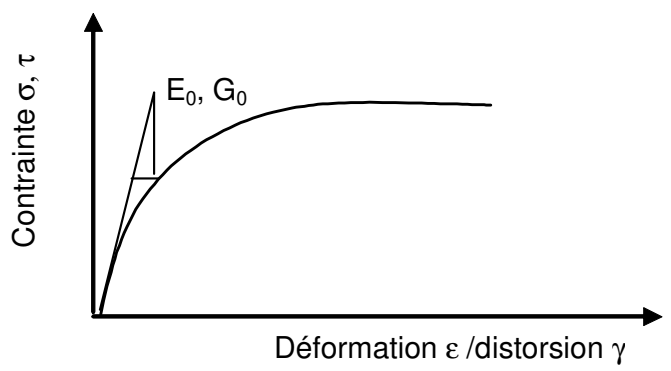

a. Courbe de contrainte-déformation du sol sous les ouvrages

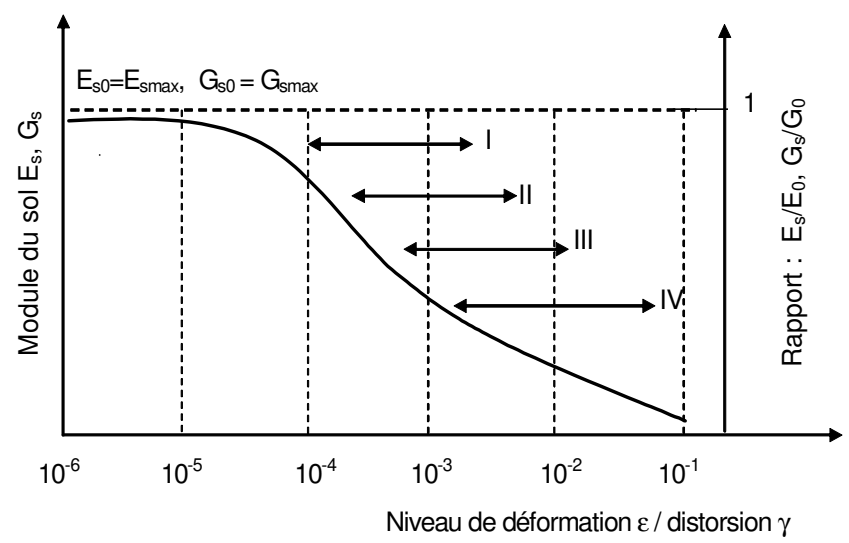

I : Ouvrages sensibles (Murs et écrans de soutènement rigides, fondations) II : Ecrans de soutènement souples

III : Tunnels et excavations souterraines

IV : Remblais sur sols mous

b. Domaine de déformation du sol en relation avec les ouvrages de construction

Figure 7 : Domaine conventionnel du niveau de déformation sous les ouvrages de construction

\section{Excavations profondes en site urbain}

En France, les calculs, le dimensionnement et les justifications des excavations profondes soutenues par des écrans de soutènement font appel à trois méthodes différentes :

- Calcul à la rupture : détermination du diagramme des pressions et mise en équations d'équilibre,

- Méthode aux coefficients de réaction: principe du ressort « unidimensionnel»,

- Calcul numérique en éléments finis : assemblage de plusieurs ressorts 2D ou 3D.
La méthode basée sur le principe du coefficient de réaction est utilisée, spécifiquement en France, de manière récurrente et elle a fait ses preuves puisque $90 \%$ des ouvrages de soutènements sont calculés, dimensionnés et justifiés par cette méthode depuis 60 ans. En effet, cette méthode permet de modéliser le sol en poussée et en butée contre l'écran de soutènement (Figure 8) par des ressorts, comme le montre la figure 9.

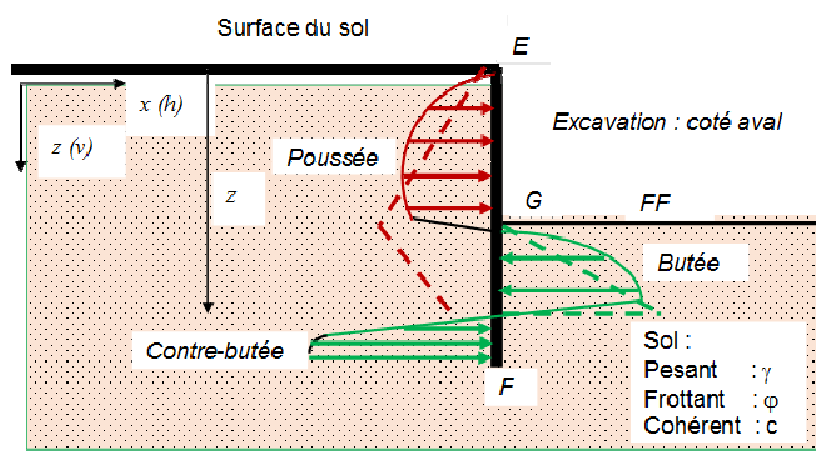

Figure 8 : Mécanisme de poussée-butée contre l'écran de soutènement

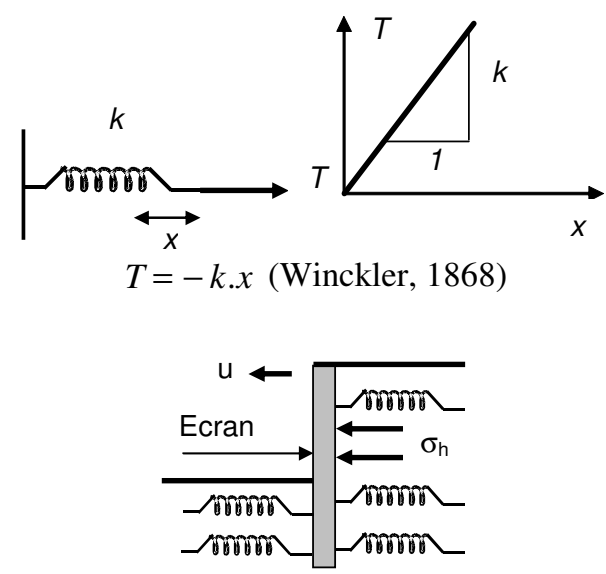

$$
p=k_{s} . u
$$

Figure 9 : Principe de la méthode du coefficient de réaction (Dhouib, 1995)

L'avantage de la méthode aux coefficients de réaction réside dans sa simplicité de mise en œuvre dans les calculs automatiques. Elle a l'inconvénient de supposer le sol «élastique » (Figure 10.a), alors qu'il en est guère (Figure 10.b). En effet, tous les codes de calculs numériques utilisant cette méthode en France sont programmés avec la courbe de réaction bilinéaire de la figure 10.a. 


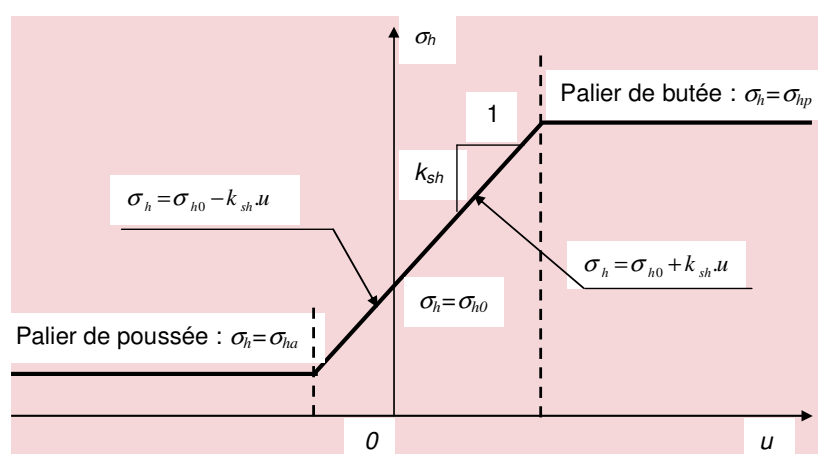

a. Courbe de réaction bilinéaire (Ménard et al., 1964)

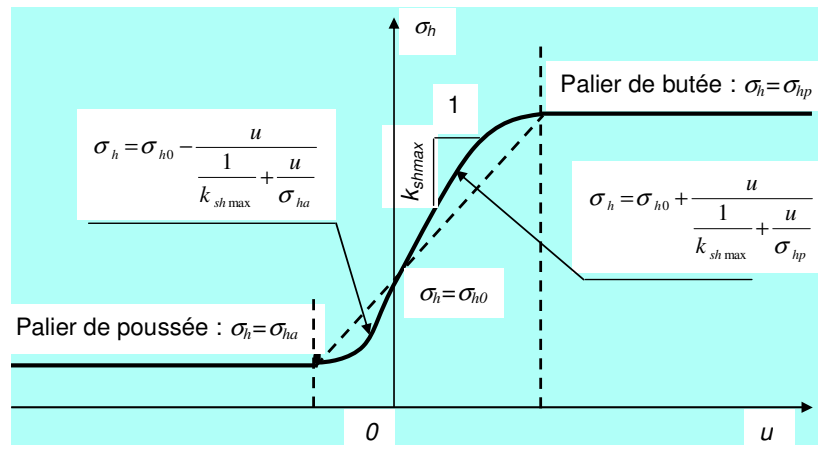

b. Courbe de réaction hyperbolique (Dhouib, 1995)

Figure 10 : Courbes de réaction pour le calcul des soutènements en France.

La figure 11 illustre le cas d'une paroi moulée ancrées par des tirants actifs.

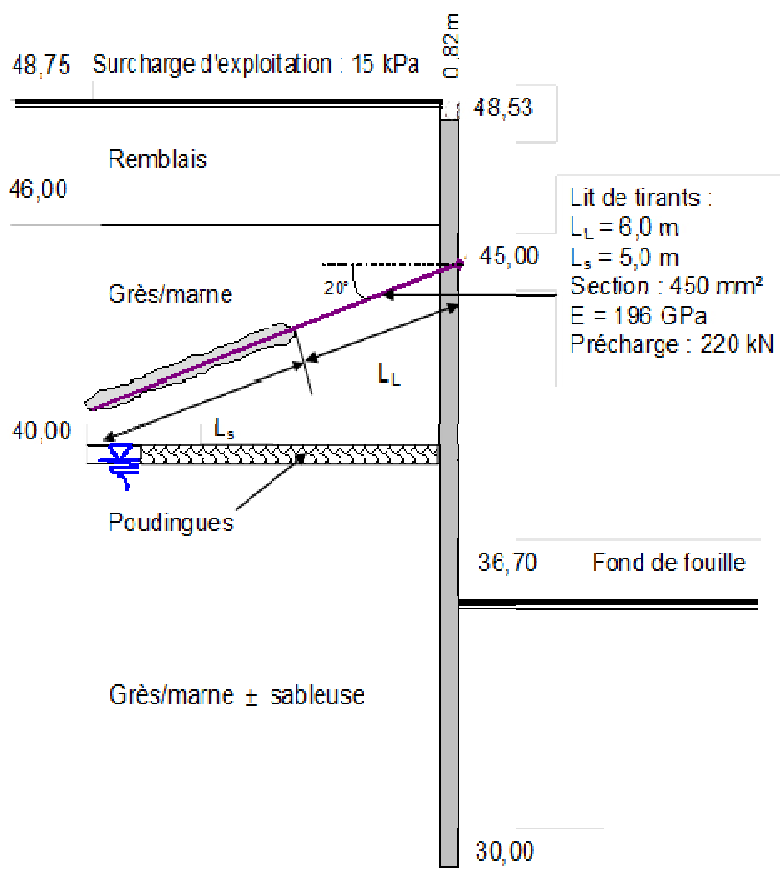

Figure 11 : Cas de paroi moulée ancrée par tirants actifs

La photo de la figure 12.a illustre le panneau de paroi instrumenté et les graphes de la figure 12.b et présentent et comparent les déplacements horizontaux mesurés aux déplacements prédits de la paroi. Les déplacements calculés en éléments finis (code «PLAXIS») et par la méthode aux coefficients de récation (logiciel «RIDO») convergent, à ces niveaux de déplacements faibles, avec les mesures (Dhouib et al., 2007).

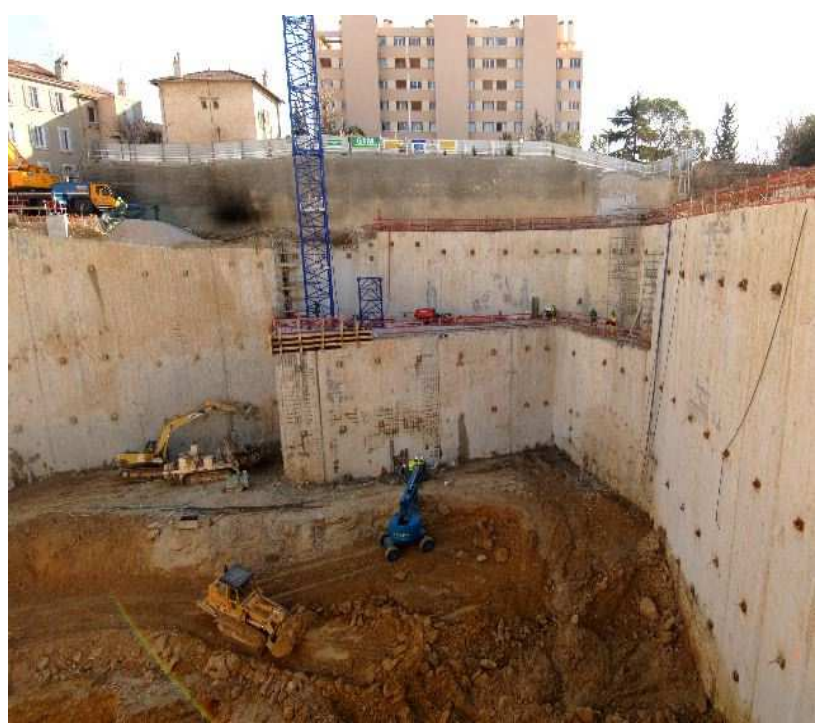

a. Elévation de la paroi instrumentée

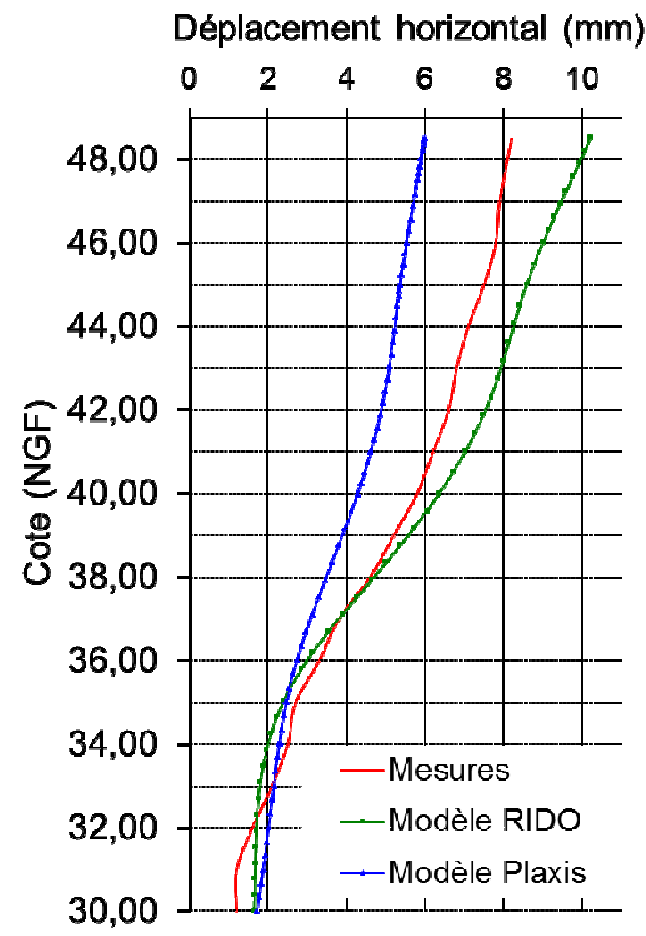

b. Comparaison des déplacements horizontaux mesurés et prédits de la poaroi

Figure 12 : Cas de paroi moulée ancrée par tirants actifs

Une autre paroi exceptionnelle d'un mégaprojet d'une grande tour de 54 étages, fouille de $70 \mathrm{~m}$ de hauteur dans un site géologiquement complexe et difficilement accessible a été instrumentée afin de suivre, d'une part, 
les déplacements de la paroi, et d'autres part, les mouvements de l'environnement immédiat.

La figure 13.a présente le site concerné par le projet et la coupe de la figure 13.b illustre la coupe type des sols et de la fouille de $70 \mathrm{~m}$ de hauteur, soutenue par trois parois différentes : micro-berlinoise en tête, puis paroi berlinoise au milieu suivie de paroi moulée pré-fondée en pied, dans laquelle vient s'incorporer la grande tour.

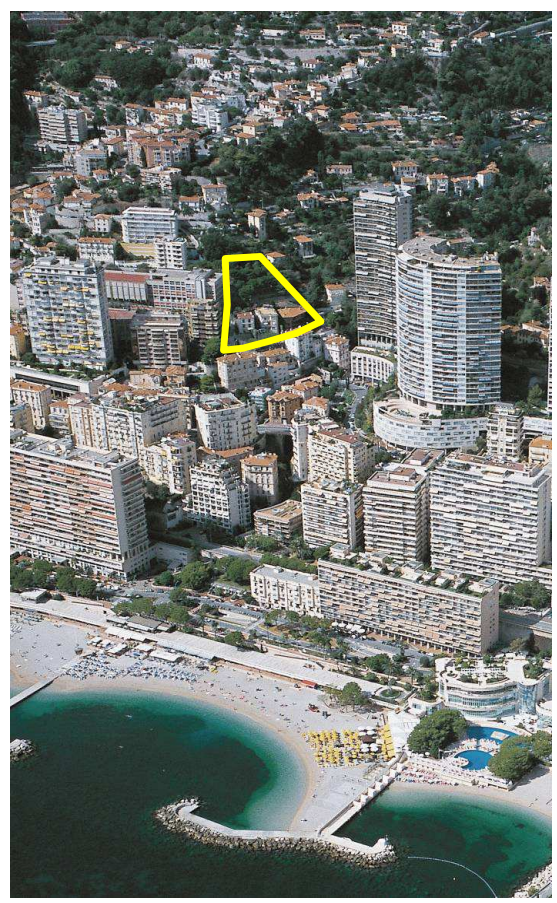

a. Site du projet de la tour

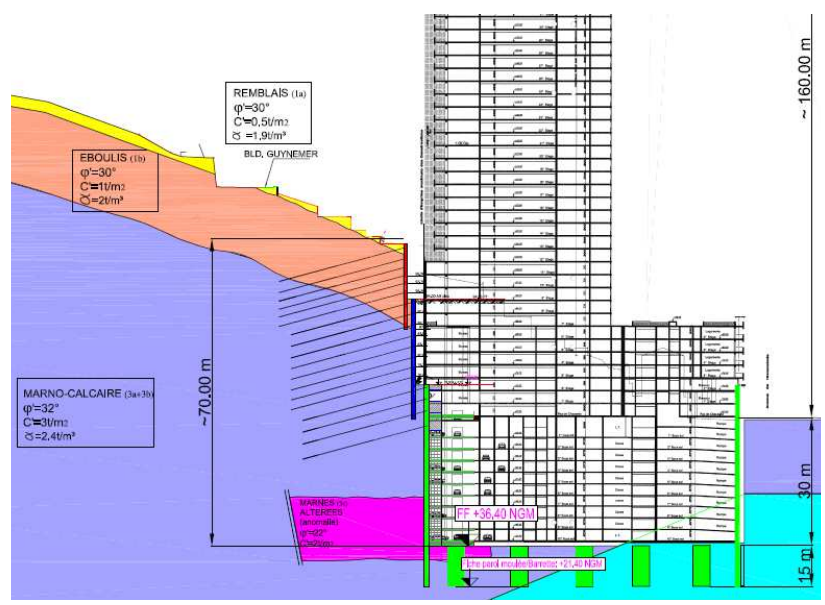

b. Coupe type de la fouille à parois multiples et de la tour de 54 étages

Figure 13 : Cas de fouilles de 70 de hauteur

La sensibilité du projet était si grande que toutes les parois ont fait l'objet de suivi des déplacements et les existants de tassements, voire de soulèvements.
La figure 14 présente les phases d'excavation et les résultats des auscultations de la paroi moulée.

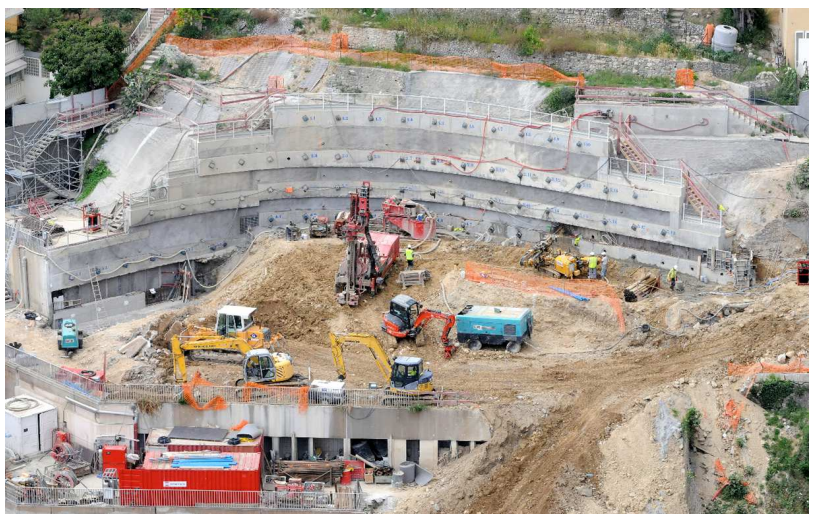

a. Paroi micro-berlinoise en cours d'excavation

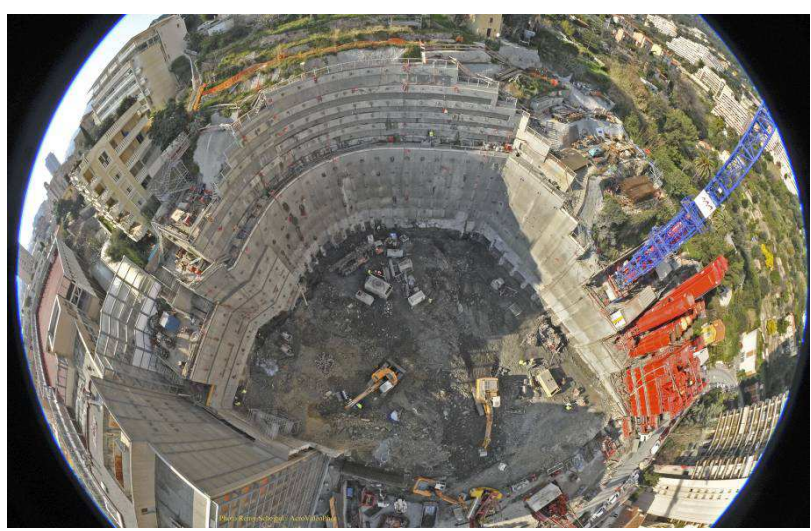

b. Fin d'excavations de la paroi berlinoise

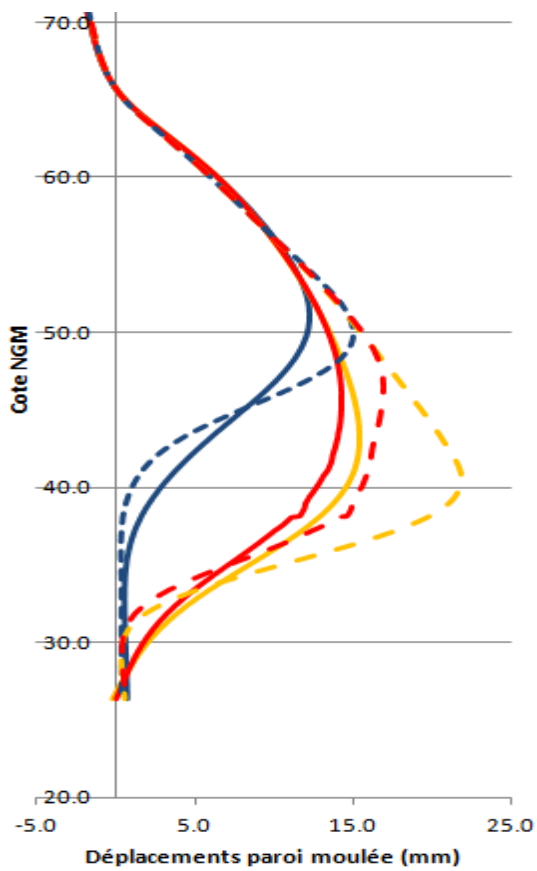

c. Déplacement en fin d'excavation (paroi moulée)

Figure 14 : Résultats des auscultations des parois

\section{Conclusions}


Les différents cas réels d'ouvrages présentés dans cette communication montrent que les déplacements des ouvrages mesurés sont toujours inférieurs aux prédictions issues des calculs, qu'ils soient analytiques ou numériques. La tendance des jeunes ingénieurs est de courir, de nos jours, aux calculs numériques car il est plus facile d'allumer son ordinateur et de lancer une «boite noire » que constituent notamment les codes en éléments finis que de prendre son crayon, du papier et la calculatrice pour avoir au moins l'ordre de grandeur des phénomènes.

Pour rendre plus crédible leur action en géotechnique, les ingénieurs doivent d'abord analyser avec critique et attention les données issues des mesures, qu'elles soient sur site ou en laboratoire, en faire une synthèse et les vérifier par rapport à des données existantes pour retenir un jeu ou plusieurs jeux de paramètres avant de se lancer dans les calculs.

Lorsque les prédictions sont faites, il est important et primordial d'élaborer un vrai programme d'auscultations et de suivi préalable sur chantier et le mettre en exécution dès le démarrage du chantier. Ce qui n'est pas toujours facile car les maitres de l'ouvrage ne veulent pas aller dans ce sens pour raisons de «fausses » économies.

Seule cette voie permettrait de confronter les prédictions à la réalité du terrain et de «promouvoir » la «Méthode observationnelle» en guise, en plus, de s'assurer de «la qualité, la solidité, la stabilité et la pérennité » des ouvrages de construction.

\section{Références bibliographiques}

1. Dhouib, A, "Méthode des coefficients de réaction appliquée en France pour les calculs de soutènements: influence de la variation des paramètres“, Revue Française de Géotechnique n ${ }^{\circ}$ 72, $3^{\text {ème }}$ trimestre 1995, pp. 11-21 (1995).

2. Dhouib, A, Lelièvre S., Idrac, ph., Betoux, O., "Station «La Blancarde» à Marseille: Comportement d'une paroi moulée à ancrages actifs", Revue Travaux n ${ }^{\circ} 844$, (Septembre 2007).

3. Dhouib, A., "Géotechnique appliquée aux projets de construction“, Presses des Ponts et Chaussées, Paris, 1041 pages (2016).

4. Ménard L., Bourdon G., Houy A., "Etude expérimentale de l'encastrement d'un rideau en fonction des caractéristiques pressiométriques du sol de fondation“, Sols-Soils n ${ }^{\circ}$ 9, pp. 11-27 (1964).

5. Ménard L., Bourdon G., "Calcul des rideaux de soutènement: Méthode nouvelle prenant en compte les conditions réelles d'encastrement ", Sols-Soils n ${ }^{\circ}$ 12, pp. 18-32 (1965).

6. Schmitt P., "Méthode des coefficients de réaction appliquée en France pour les calculs de soutènements: influence de la variation des paramètres", Revue Française de Géotechnique n ${ }^{\circ}$ 72, $3^{\text {ème }}$ trimestre 1995, pp. 11-21 (1995).

7. Winkler E., "Die Lehre von der Elasticitäs und Festigkeit“, Prag. Verlag H. Dominicus (1868). 